\title{
ANÁLISIS DE CORRELACIÓN Y REGRESIÓN PARA LA DETERMINACIÓN DEL NITRÓGENO EN MAÍZ
}

\author{
Nevio A. Bonilla Morales'
}

\section{RESUMEN}

\begin{abstract}
Análisis de correlación y regresión para la determinación del nitrógeno en maíz.
El objetivo fue identificar la relación existente entre el contenido del nitrógeno foliar determinado en laboratorio con el medido en el campo mediante un clorofilómetro. Se utilizó un diseño de bloques completos al azar con arreglo factorial de cuatro variedades de maíz, tres repeticiones y cuatro niveles de nitrógeno. Las variedades de maíz fueron UPIAV-G6, Los Diamantes 8843, JSAENZ y EJN2 y los niveles de nitrógeno 0, 60, 120 y $180 \mathrm{~kg}$ nitrógeno/ha. Los niveles de nitrógeno para variedades mostraron valores de 0,39 a 0,62 en los modelos de regresión. Los coeficientes de correlación entre contenido foliar en laboratorio y las lecturas del medidor de clorofila mostraron un valor de 0,71 con referencia al factor variedad y 0,55 con respecto a nivel de nitrógeno. Los contenidos de nitrógeno por variedad variaron entre 2,63 y $3,41 \%$ y para los niveles de nitrógeno entre 2,66 y $2,76 \%$. El coeficiente de correlación de Pearson varió de 0,40 a 0,62 con el muestreo foliar y las lecturas de campo lo que indica una relación importante entre ambos factores.
\end{abstract}

Palabras clave: medidor de clorofila, regresión, correlación, Zea mays.

\section{INTRODUCCION}

La producción de maíz en Costa Rica se desarrolla en diversidad de ambientes que provocan en muchos casos bajos rendimientos. Los principales factores que afectan su producción son la fertilidad y el manejo de los suelos, la distribución de las lluvias, el inadecuado manejo agronómico y la presencia de enfermedades y plagas.

Los trabajos de investigación en fertilidad del cultivo de maíz presentan ciertas complejidades, ya que consideran aspectos de densidad de siembra, cultivares, rotación con leguminosas y épocas de siembra. De igual manera, los trabajos llevados a cabo por Saín y Acosta (1993), Barreto et al. (1994), Gordon et al. (1997), Tosquy y Castañon (1998), Morales (1998) y Cano et al. (2001) muestran resultados interesantes al respecto. Se reportan interacciones entre densidad de siembra, épocas de siembra y cultivares; así también la dinámica de la rotación maíz-frijol en función del manejo de la fertilización.
En los últimos años han surgido herramientas metodológicas que determinan las dosis de fertilización nitrogenada adecuada para el cultivo de maíz. Se han utilizado de manera combinada los experimentos clásicos de niveles del elemento con diferentes cultivares con la determinación indirecta del contenido de clorofila mediante el medidor de clorofila SPAD y la medición en laboratorio del contenido foliar de nitrógeno utilizando el método Kjeldahl (AOAC 1984, Ma y Zuazaga 1942). Existe una gran diversidad de variantes con respecto a esta metodología, dependiendo de los objetivos del trabajo y el cultivo de que se trate. MacKown y Sutton (1998) llevaron a cabo experimentos con esta metodología en tabaco; Rodríguez et al. (1998) en tomate; Peterson et al. (1993), Novoa y Villagrán (2002), Sainz Rozas y Echeverría (1998) en maíz. De esta manera, determinaron los niveles de nitrógeno a través de la medición del verdor de las hojas con el SPAD y su correlación con los contenidos reales de nitrógeno foliar (Rodríguez et al. 1998, Peterson et al. 1993, Novoa y Villagrán 2002, Sainz Rozas y Echeverría 1998).

\footnotetext{
${ }^{1}$ Instituto Nacional de Innovación y Transferencia en Tecnología Agropecuaria, INTA. Costa Rica. nbonilla@ inta.go.cr
} 
Las mediciones de clorofila realizadas con el SPAD y el análisis de contenido de nitrógeno están altamente correlacionados (Novoa y Villagrán 2002), donde se reporta un coeficiente de regresión de 0,88 y 0,81, respectivamente.

En este caso, se menciona que para valores del SPAD inferiores a 35,3, equivalentes a un contenido de $1,84 \%$ de $\mathrm{N}$ en las hojas, estarían indicando que es necesario aplicar nitrógeno, considerando el valor crítico del elemento.

EISPAD ó medidor de clorofila mide el contenido de clorofila "o verdor" de las plantas, cuantifica cambios sutiles o tendencias en el contenido nutricional de la planta mucho antes de que ellos sean visibles al ojo humano y permite determinar el estado del nitrógeno en las hojas del cultivo. Es una medición no invasiva, ni destructiva del tejido foliar; simplemente determina un contenido de clorofila incluido en un índice con una lectura de valor absoluto que va de 0 a 99,9. La relación entre la lectura del medidor de clorofila y la concentración de clorofila foliar no es lineal, por lo tanto el uso básico del medidor ha sido para determinar la eficacia potencial de los tratamientos adicionales de nitrógeno en las plantas de un cultivo (Markwell et al. 1995).

El medidor de clorofila constituye una herramienta apropiada para el monitoreo de la disponibilidad de $\mathrm{N}$, especialmente en estadios vegetativos del cultivo. Sainz Rozas y Echeverría (1998) indican que cuando los datos de los dos años del estudio fueron analizados, el 95\% del rendimiento máximo fue logrado con índice de suficiencia de nitrógeno (ISN) de 0,97-0,98.

En el cultivo de maíz dulce este dispositivo se considera un buen estimador del estado del nitrógeno en la planta y podría usarse como complemento de los programas de fertilización, ya que sus lecturas se relacionan directamente con el contenido de nitrógeno en las hojas y con el rendimiento del cultivo (Villar 2004). Similares resultados obtuvieron MacKown y Sutton (1998) en tabaco, Rodríguez et al.
(1998) en tomate y Arregui et al. (2000) en papa. Sin embargo, se recomienda validar el modelo en una segunda oportunidad, pues los resultados obtenidos en otros estudios no muestran los datos necesarios para poder inferir que la dosis estimada por el modelo fue la que produjo los resultados encontrados y no un factor ajeno. Villar (2004) determinó que el valor crítico calculado para lectura de clorofila 49 , permitiría contar con una herramienta en la toma de decisiones respecto a la aplicación de nitrógeno en la segunda fertilización. Este valor crítico en conjunto con la utilización del modelo, servirían para proporcionar recomendaciones de dosis de fertilizante a aplicar.

El objetivo fue identificar la relación existente entre el contenido del nitrógeno foliar determinado en laboratorio con el medido en el campo mediante un clorofilómetro.

\section{MATERIALES Y MÉTODOS}

Esta investigación se estableció en las localidades de Pejibaye de Pérez Zeledón y Upala de Alajuela en las dos épocas de siembra para cada localidad (mayo-agosto y setiembre-diciembre) de los años 2007-2008.

La actividad se desarrolló en dos etapas paralelas:

1. Establecimiento y evaluación de los tratamientos con niveles de nitrógeno y variedades de maíz bajo las condiciones de producción del productor de maíz de los cantones de Pejibaye de Pérez Zeledón y Upala en dos épocas de siembra.

2. Determinación del contenido de nitrógeno foliar, en el laboratorio de suelos del INTA, de las muestras tomadas en cada ensayo durante el desarrollo del ciclo del cultivo.

Las parcelas experimentales estuvieron constituidas por cuatro surcos de $5,0 \mathrm{~m}$ de longitud separados $0,75 \mathrm{~m}$. La parcela útil consideró los dos surcos centrales con un área de $8,25 \mathrm{~m}^{2}$. Las plantas estuvieron distanciadas $0,5 \mathrm{~m}$ entre sí, se sembraron tres semillas por 
golpe de siembra, se raleó a dos plantas por golpe de siembra a los 15 días después de la siembra, para tener una población homogénea y un número constante de plantas durante todo el ciclo del cultivo. La población fue de 53 333 plantas por hectárea.

A partir de la etapa fenológica V6 (seis hojas verdaderas) se muestreó cada 15 días hasta el final del ciclo del cultivo, seleccionando la primera hoja totalmente desarrollada ubicada desde el extremo superior de la planta hacia abajo, esto se llevó a cabo en tres plantas ubicadas en cada borde de la parcela para no afectar la determinación del rendimiento de las mismas en los surcos de la parcela útil. Se realizaron 16 mediciones con el medidor de clorofila en cada hoja muestreada antes de ser cosechada, cada muestra se llevó al laboratorio para la determinación del contenido foliar de nitrógeno mediante el método Microkjeldahl (AOAC 1984, Ma y Zuazaga 1942).

El nitrógeno se aplicó un $25 \%$ a la siembra y un $75 \%$ a los 25 días después de la siembra. Solamente los tratamientos con 0 nitrógeno no llevaron este elemento. El fósforo y el potasio se aplicaron en un $100 \%$ al momento de la siembra. La fuente de nitrógeno fue la fórmula química comercial $10-30-10$ a la siembra y nitrato de amonio a los 25 días después de la siembra.

\section{Variables evaluadas (CIMMYT 1995)}

\section{En campo}

1. Número de plantas establecidas (a los 15 días de siembra).

2. Contenido de clorofila cada 15 días a partir de etapa V6 hasta el final del ciclo.

3. Días a floración masculina (número de días con $50 \%$ de plantas floreadas).

4. Días a floración femenina (número de días con $50 \%$ de plantas floreadas).

5. Altura de planta (en centímetros al momento de la cosecha).

6. Altura de mazorca (en centímetros al momento de la cosecha).

7. Acame de raíz (número de plantas).
8. Acame de tallo (número de plantas).

9. Daño por enfermedades foliares (escala 1 a 5 donde 1 es sano).

10. Daño por insectos plaga (escala 1 a 5 donde 1 es sano).

11. Aspecto de planta (escala donde 1 es excelente y 5 deficiente).

12. Aspecto de mazorca (escala donde 1 es excelente).

13. Cobertura de mazorca (número de mazorcas con mala cobertura).

14. Número de plantas cosechadas.

15. Número de mazorcas podridas (al momento de la cosecha).

16. Peso de campo (en kilogramos por parcela útil).

17. Humedad de grano a cosecha (porcentaje medido con determinador portátil).

\section{En laboratorio}

Contenido foliar de nitrógeno (determinado con método micro-Kjeldahl). Micro Kejldahl (AOAC 1984, Ma y Zuazaga 1942). De acuerdo con Vargas (2005) se utilizó el método Microkjeldahl que consta de tres etapas, a saber, digestión, destilación y valoración para la determinación del contenido de nitrógeno foliar de las muestras colectadas de los experimentos de campo establecidos.

Se realizó un análisis de suelo de cada sitio de siembra previo al establecimiento del ensayo.

\section{Análisis estadístico}

Se realizó un análisis de regresión lineal para los niveles de nitrógeno en el tiempo para cada variedad, un análisis de correlación entre lecturas de SPAD y el contenido foliar de nitrógeno del laboratorio obtenido por el método de Micro Kejldahl (AOAC 1984, Ma y Zuazaga 1942). Las otras variables se registraron como referencia y complemento del rendimiento.

El análisis de la varianza para la primera variable se realizó mediante el procedimiento GLM del programa Statistical Analysis System (SAS) (SAS Institute Inc. 1985). Se utilizó la 
prueba de separación de medias Diferencia Mínima Significativa (DMS). Además, se calculó el coeficiente de correlación de Pearson y su significancia para validar la capacidad estimadora del SPAD.

Los modelos de regresión también fueron ajustados mediante el mismo programa, las medias de tratamientos fueron comparadas mediante contrastes ortogonales para determinar si las necesidades de nitrógeno por parte de la planta de maíz presentan diferencias varietales. En este caso se supuso que las variedades no son igualmente exigentes en los niveles por este elemento. En este artículo se presentan los resultados combinados de dos años de evaluación (2007 y 2008) correspondientes a cuatro ensayos ubicados en las localidades de Upala y Pejibaye de Pérez Zeledón.

\section{RESULTADOS Y DISCUSIÓN}

El análisis de regresión indicó que el modelo seleccionado que considera los factores variedad y nivel de nitrógeno explica el $74,0 \%$ de la varianza de los datos. La ecuación de regresión es la siguiente:
$Y=2,346+0,291$ (variedad) $+0,0154$ (nivel nitrógeno)

El análisis de contrastes determinó significancia para él para el modelo de regresión lineal al 0,05 en cuanto a los factores nivel de nitrógeno y variedad.

El análisis de regresión para la variable dependiente rendimiento mostró que el modelo es significativo con un coeficiente de determinación de 0,99. Esto indica que para determinado nivel de nitrógeno el modelo de regresión predice adecuadamente el rendimiento.

El análisis de regresión evidenció significancia para el modelo de regresión lineal al 0,05 en cuanto a los factores nivel de nitrógeno y variedad. El coeficiente de determinación indicó un valor 0,84 , lo que indica una adecuada explicación de la variabilidad de los datos.

Las predicciones a partir del modelo de regresión en el análisis de varianza acumulado con respecto a variedades y niveles de nitrógeno para la variable rendimiento presentaron una significancia para el modelo considerado (Cuadro 1).

Cuadro 1. Predicciones de los valores de rendimiento para variedades y niveles de nitrógeno del modelo de regresión. San José, CR. 2007.

\begin{tabular}{|cc|}
\hline Variedad & Valor de rendimiento predicho \\
EJN2 & 4,344 \\
JSAENZ & 3,753 \\
LD8843 & 4,703 \\
UPIAVG6 & 5.126 \\
\hline Nivel de Nitrógeno & Valor de rendimiento predicho \\
0 & 3,352 \\
60 & 3,629 \\
120 & 4,833 \\
180 & 6,133 \\
\hline
\end{tabular}

El análisis de regresión indicó para las variedades EN2, LD8843, JSAENZ y UPIAV-G6 porcentajes de variancia de $71,3,86,4,94,5$ y 89,5 respectivamente. Esto indica que el modelo de regresión explica bastante bien la variabilidad de los datos. Así mismo en cuanto los niveles de nitrógeno el coeficiente de determinación fue de 0,98 para el modelo de regresión ajustado con una significancia al 0,05 . En la Figura 4 se muestra la línea de mejor ajuste para el modelo de regresión de la variable rendimiento según el nivel de nitrógeno. 
El análisis de regresión lineal para los niveles de nitrógeno para cada variedad mostró valores desde 0,39 a 0,62 en los modelos de regresión indicando que en las variedades una relativa respuesta lineal a la aplicación del elemento.

Los valores de correlación fueron altos entre ambas variables a través de las diferentes etapas fenológicas de la planta de maíz, esta tendencia se muestra tanto para los tratamientos de nitrógeno como para las diferentes variedades. Además, se calculó el coeficiente de Pearson para determinar la capacidad estimadora del SPAD, del Cuadro 2 se evidencia que esta capacidad es relativamente alta entre las lecturas de SPAD y los valores de nitrógeno determinados en laboratorio.

Dicha tendencia se muestra también en la Figura 1, donde se presenta la misma para las diferentes variedades en función del rendimiento y las lecturas de SPAD, siendo la variedad UPIAV-G6 la que presenta la más clara. Esto evidencia que esta variedad responde mejor a los tratamientos de nitrógeno. La variedad LD 8843 también presenta una tendencia similar. En el Cuadro 2 se presentan las correlaciones entre las lecturas de SPAD y el porcentaje de nitrógeno determinado en laboratorio para los ensayos de 2007.
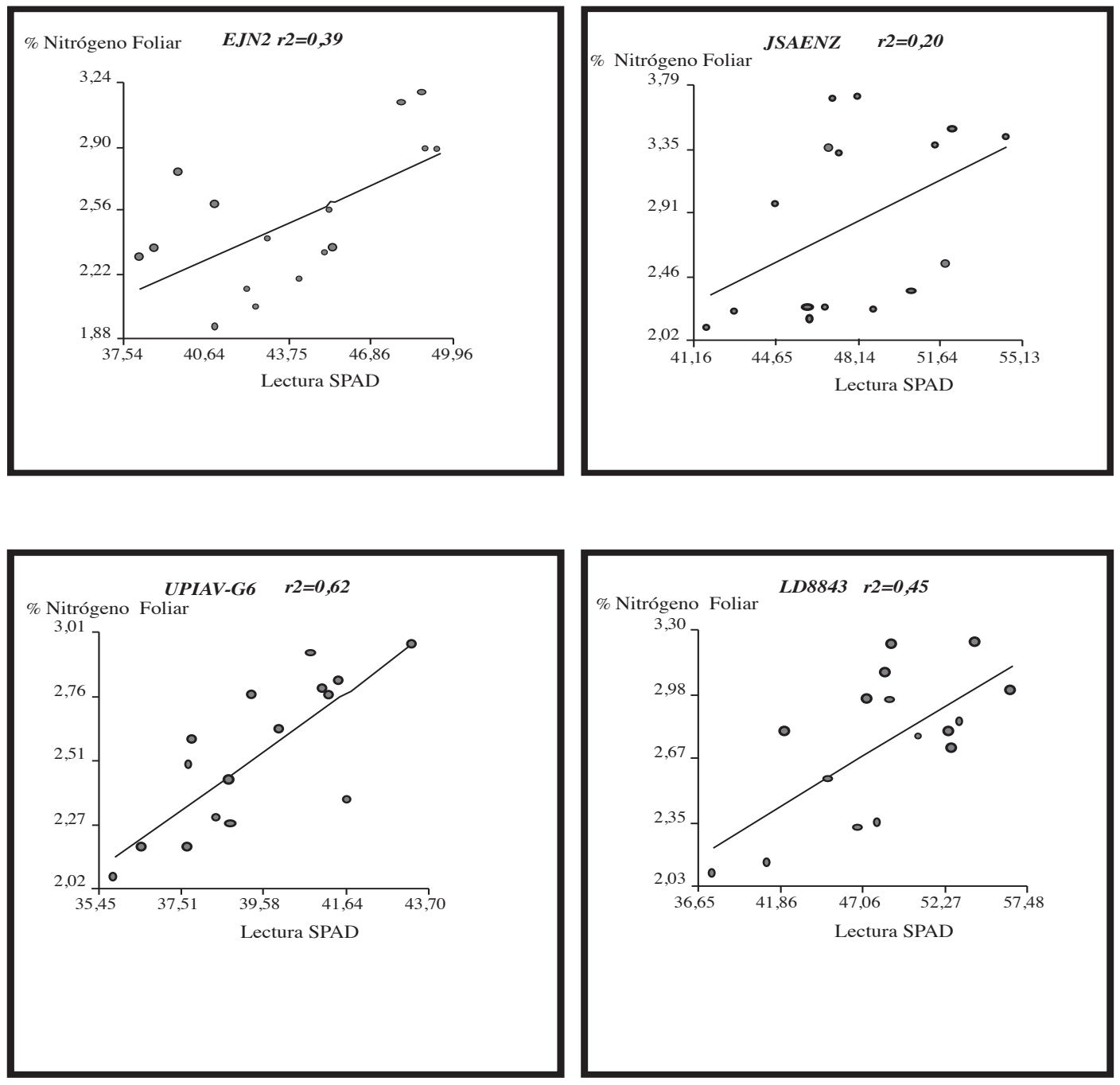

Figura 1. Correlación entre lecturas de SPAD y rendimiento de grano en $\mathrm{t} /$ ha para las cuatro variedades de maíz. Pejibaye, Pérez Zeledón, CR. 2007. 
Cuadro 2. Correlaciones entre lecturas de SPAD y porcentaje de nitrógeno en diferentes etapas fenológicas de la planta de maíz según tratamiento y variedad. El Águila, Pejibaye. San José, CR. 2007.

\begin{tabular}{|c|c|c|c|c|c|c|c|c|c|c|}
\hline & \multicolumn{2}{|c|}{$21 / 06 / 07$} & \multicolumn{2}{|c|}{$04 / 0707$} & \multicolumn{2}{|c|}{ 19/7/07 } & \multicolumn{2}{|c|}{ 09/08/07 } & \multicolumn{2}{|c|}{ Promedio } \\
\hline VARIEDAD & SPAD & $\% \mathbf{N}$ & SPAD & $\% \mathbf{N}$ & SPAD & $\% \mathbf{N}$ & SPAD & $\% \mathrm{~N}$ & SPAD & $\% \mathrm{~N}$ \\
\hline UPIAV-G6 & 46,6 & 3,27 & 48,3 & 2,76 & 50,4 & 3,14 & 43,3 & 2,45 & 47,1 & 2,91 \\
\hline LD8843 & 45,1 & 2,94 & 47,0 & 2,45 & 49,3 & 3,05 & 42,4 & 2,31 & 45,9 & 2,69 \\
\hline JSAENZ & 44,3 & 2,74 & 44,5 & 2,39 & 47,5 & 3,02 & 40,9 & 2,37 & 44,3 & 2,63 \\
\hline EJN2 & 42,9 & 3,10 & 45,3 & 2,50 & 49,6 & 3,15 & 44,4 & 2,52 & 45,6 & 2,82 \\
\hline $\mathbf{R}$ & & 0,39 & & 0,82 & & 0,84 & & 0,78 & & 0,71 \\
\hline 0 & 44,0 & 2,90 & 45,3 & 2,60 & 49,6 & 2,90 & 41,3 & 2,30 & 45,0 & 2,66 \\
\hline 60 & 44,0 & 2,90 & 46,1 & 2,60 & 50,6 & 2,90 & 43,6 & 2,40 & 46,1 & 2,70 \\
\hline 120 & 45,1 & 3,20 & 46,8 & 2,40 & 50,0 & 3,00 & 41,9 & 2,40 & 46,0 & 2,76 \\
\hline 180 & 45,7 & 3,00 & 47,0 & 2,60 & 48,9 & 3,00 & 41,7 & 2,30 & 45,8 & 2,73 \\
\hline $\mathbf{R}$ & & 0,62 & & 0,49 & & 0,71 & & 0,44 & & 0,55 \\
\hline r Pearson & & 0,30 & & 0,45 & & 0,48 & & 0,62 & & \\
\hline
\end{tabular}

Las correlaciones entre las lecturas de SPAD y el porcentaje de nitrógeno se presentan en la Figura 2 para el ensayo de Upala y la Figura 3 en el caso del ensayo establecido en Pejibaye. Al analizar los gráficos mencionados se nota que las correlaciones son relativamente altas para ambos casos. Dichas correlaciones son particularmente interesantes para las variedades UPIAV-G6 y Los Diamantes 8843 en ambos ensayos.

Los coeficientes de correlación entre las factores y variables consideradas para el ensayo de Upala fueron \% nitrógeno y SPAD: 0,43, SPAD y nivel nitrógeno: 0,53, rendimiento y SPAD: 0,50, rendimiento y nivel de nitrógeno: 0,39, rendimiento y \% nitrógeno: 0,006. Esto indica que el medidor de clorofila correlacionó aceptablemente con la mayor parte de las variables (Figuras 2 y 3 ).

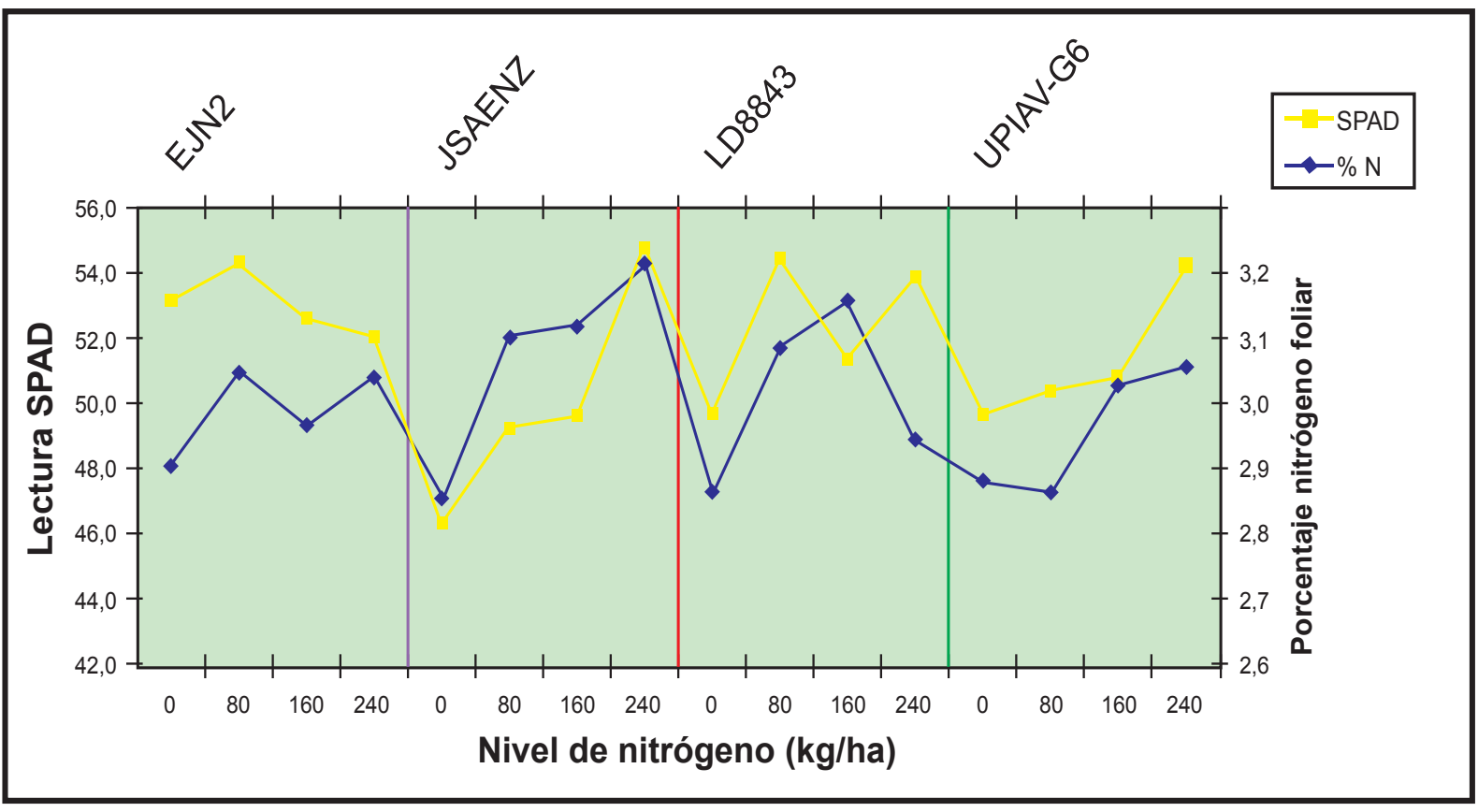

Figura 2. Relación de lectura SPAD y porcentaje de nitrógeno foliar para las variedades estudiadas. Upala, Alajuela, CR. 2008. 


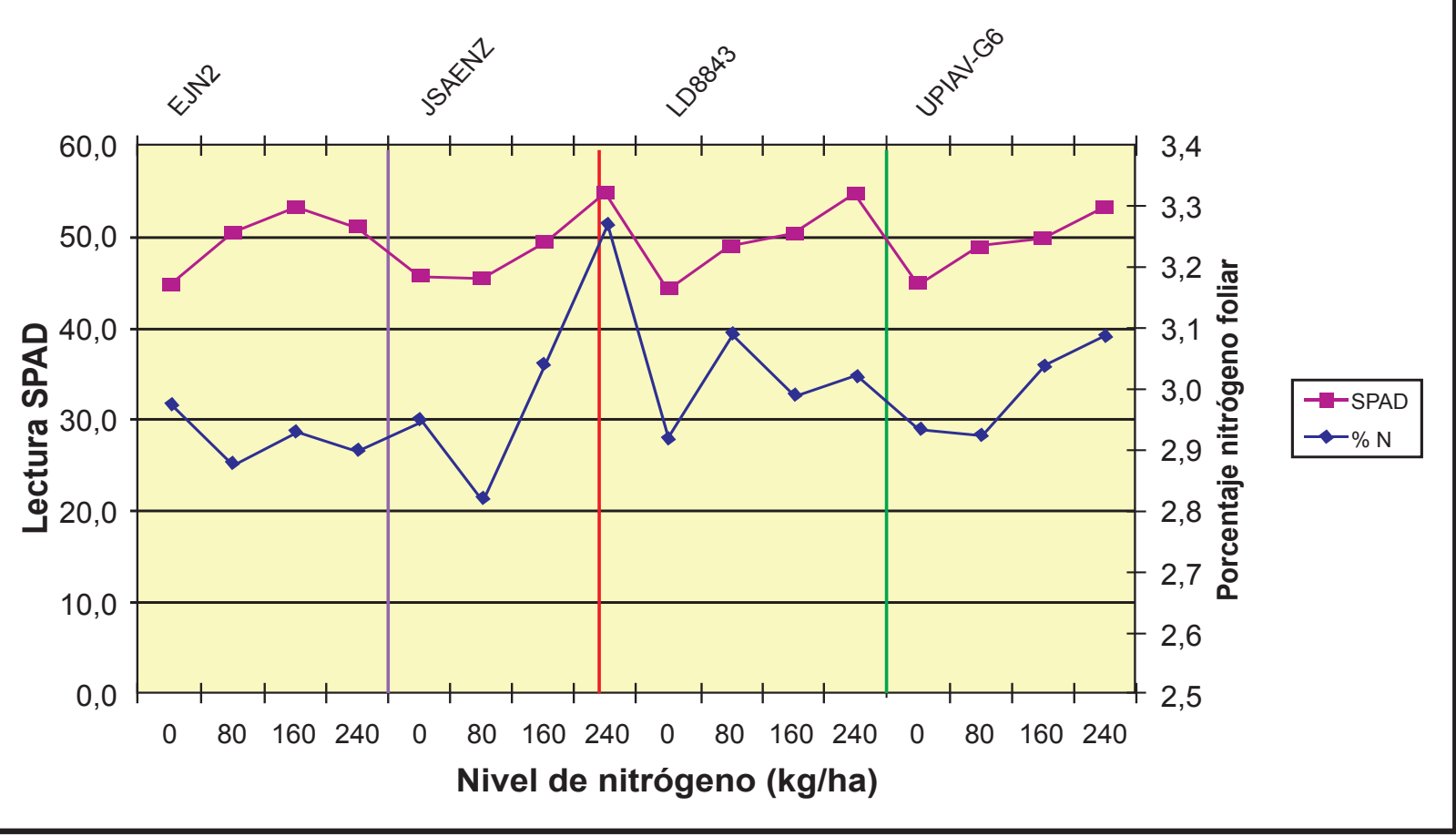

Figura 3. Relación de lectura SPAD y porcentaje de nitrógeno foliar para las variedades estudiadas. Pejibaye, Pérez Zeledón, CR. 2008.

En el Cuadro 3 se muestran los porcentajes de nitrógeno al momento de la cosecha en mazorca para los ensayos de 2008 tanto para los tratamientos de nitrógeno como las variedades consideradas. Es importante indicar que los valores de nitrógeno son bajos con respeto a lo mostrado durante el ciclo del cultivo como una consecuencia de la transformación de este elemento en moléculas más complejos en la mazorca de maíz.

Los coeficientes de correlación para el ensayo de Pejibaye fueron porcentaje de nitrógeno y SPAD: $0,54 \%$ nitrógeno y rendimiento: 0,077; SPAD y rendimiento: 0,43 ; rendimiento y nivel de nitrógeno: 0,47; SPAD y nivel de nitrógeno: 0,90. Estos resultados conservan una tendencia similar al ensayo de Upala con respecto al SPAD.

No se determinaron diferencias significativas en el ensayo de Upala en cuanto a niveles de nitrógeno y variedades con respecto a rendimiento. Para el ensayo de Pejibaye si se determinaron diferencias significativas para los dos factores mencionados. 
Cuadro 3. Porcentaje de nitrógeno a la cosecha para las localidades de Upala y Pejibaye según nivel de nitrógeno y variedad de maíz. Alajuela, CR. 2008.

\begin{tabular}{|cccc|}
\hline VARIEDAD & NIVEL N & Upala $\% \mathbf{N}$ & Pejibaye $\% \mathbf{~ N}$ \\
\hline EJN2 & 0 & 1,8 & 1,7 \\
& 60 & 1,9 & 1,4 \\
& 120 & 1,9 & 1,5 \\
JSAENZ & 180 & 1,9 & 1,8 \\
& 0 & 1,9 & 1,6 \\
& 60 & 1,9 & 1,6 \\
LD8843 & 120 & 1,9 & 1,7 \\
& 180 & 2,0 & 1,6 \\
& 0 & 1,8 & 1,6 \\
& 60 & 2,0 & 1,6 \\
UPIAV-G6 & 120 & 1,8 & 1,6 \\
& 180 & 1,9 & 1,6 \\
& 0 & 1,9 & 1,5 \\
& 60 & 1,8 & 1,6 \\
& 120 & 1,9 & 1,5 \\
& 180 & 1,9 & 1,5 \\
\hline
\end{tabular}

De acuerdo a los resultados obtenidos, se puede señalar que los coeficientes de correlación entre contenido foliar determinado en laboratorio y las lecturas del medidor de clorofila mostraron un valor de 0,71 con referencia al factor variedad y 0,55 con respecto a nivel de nitrógeno lo que evidencia una correlación relativamente aceptable entre ambos métodos de determinación. Los contenidos de nitrógeno por variedad oscilaron entre 2,63 y 2,91 \% y para los niveles de nitrógeno variaron entre 2,66 y $2,76 \%$. Estos son valores relativamente normales para el cultivo de maíz El coeficiente de correlación de Pearson varió de 0,30 a 0,62 a lo largo del tiempo de muestreo foliar y toma de lecturas con el SPAD lo que valida la capacidad estimadora del medidor de clorofila indicando que la correlación varía dependiendo de la variedad y el nivel de nitrógeno de que se trate.

\section{Rendimiento de grano (t/ha)}

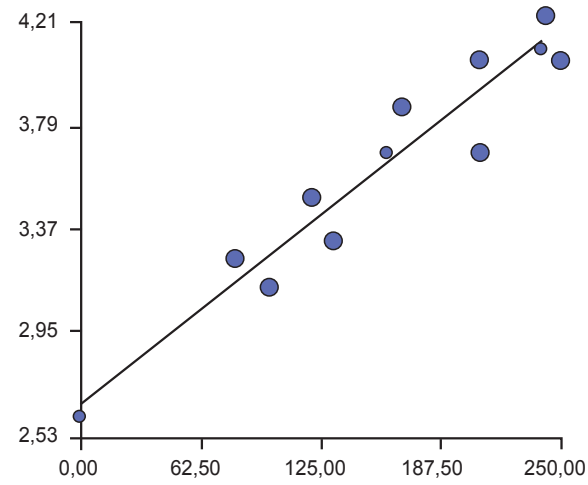

Nivel de nitrógeno (kg/ha)

Figura 4. Función de regresión lineal para rendimiento según nivel de nitrógeno. CR. 2011 


\section{LITERATURA CITADA}

AOAC (Association Of Official Analytical Chemists). 1984. Official methods of analysis. 13Th. Ed. Washington, D.C. 1298 p.

Arregui, I.M.; Merina M.; Mingo-Castel, A.M. 2000. Aplicación del medidor portátil de clorofila en los programas de fertilización nitrogenada en patata de siembra Depto. de Producción Agraria, Universidad Pública de Navarra. In. Pascualena J, Ritter E. (Ed) 2000. Libro de Actas del Congreso Iberoamericano de Investigación y Desarrollo en Patata. Patata. 3-6 Julio, Vitoria-Gastéis, España. p 157-170.

Barreto, H. J.; Pérez, C.; Fuentes, M.R.; Quemé, J.L.; Larios, L. 1994. Efecto de dosis de urea- $\mathrm{N}$ en el rendimiento del maíz bajo un sistema de rotación con leguminosas de cobertura. Agronomía Mesoamericana 5:8895.

Cano, O.; Tosquy, O.H.; Sierra, M.; Rodríguez, F.A. 2001 Fertilización y densidad de población en genotipos de maíz cultivados bajo condiciones de temporal. Agronomía Mesoamericana 12(2):199-203.

CIMMYT (Centro Internacional de Mejoramiento en Maíz y Trigo). 1995. Manejo de los ensayos e informe de los datos para el Programa de Ensayos Internacionales de Maíz del CIMMYT. México, D.F. 20p.

García, F.O. 2003. Criterios para el manejo de la fertilización del cultivo de maíz INPOFOS/ PPI/PPIC Cono Sur Acassuso - Argentina. 21 p.

Gordón, R.; Franco, J.; De Gracia, N.; González, A. 1997. Respuesta del maíz al nitrógeno y la rotación con canavalia, bajo dos tipos de labranza, Río Hato, Panamá, 199394. Agronomía Mesoamericana 8(2):78-84.

Hernández, A; Barrientos, V; Chassaigne, A; Alezones, J. 2003. Evaluación y selección de poblaciones y líneas de maíz (Zea mays
L.) eficientes en la asimilación de nitrógeno. Bioagro 15(2):115-120.

Ma, T. S.; Zuazaga, G. 1942.Micro-Kjeldahl determination of nitrogen. A new indicator and an improved rapid method. Ind. Eng. Chem. (Analytical Edition) 14:280-2, [G.H. Jones Chemical Laboratory, University of Chicago, Chicago, IL]

Mackown, C.T.; Sutton, T.G. 1998 Using Early-Season Leaf Traits to Predict Nitrogen Sufficiency of Burley Tobacco Agron. J. 90:2127.

Markwell , J; Osterman, J.C.; Mitchell, J.L. 1995. Calibration of the Minolta SPAD-502 leaf chlorophyll meter Photosynthesis Research 46 (3): 467-472

Morales, J.A. 1998. Fertilización y densidad de población en líneas de maíz en el noreste de México. Agronomía Mesoamericana 9 (2):125130.

Novoa, R.; Villagrán, N. 2002 Evaluación de un instrumento medidor de clorofila en la determinación de niveles de nitrógeno foliar en maíz .Agric. Téc. 62(1) Chillán ene., sp.

Peterson, T.A.; Blackmer, T; Francis, D; Schepers, J. 1993. NebGuide: Using a Chlorophyll Meter to Improve N Management. Cooperative Extension, Institute of Agriculture and Natural Resources, University of Nebraska. Lincoln. EUA No.G93-1171-A. 4p.

Rodríguez Mendoza, M; González, G; Santelises, A; Etchevers Barra, J.; Santizó Rincón, J. A. 1998. Estimación de la concentración de nitrógeno y clorofila en tomate mediante un medidor portátil de clorofila Instituto de Recursos Naturales, Colegio de Postgraduados. México. Terra 6(2):135-141.

Saín, G.; Acosta M. A. 1993 Recomendaciones condicionadas de fertilización con nitrógeno y fósforo usando un modelo cuadrático en la provincia de Chiriquí, Panamá. Agronomía Mesoamericana 4:11-17. 
Sainz Rozas, H.; Echeverría, H. 1998. Relación entre las lecturas del medidor de clorofila (Minolta SPAD 502) en distintos estadios del ciclo del cultivo de maíz y el rendimiento en grano. Rev. Fac. Agron., La Plata 103(1):3744.

Sainz Rozas, H; Echeverría, H.E; Barbieri, P. 2004. Desnitrificación en un suelo bajo siembra directa en función de la presencia de plantas de maíz y de la dosis de nitrógeno. Revista de la Asociación Argentina de la Ciencia del Suelo. Ciencia del Suelo 22(1): 27-35.

Tosquy, H.; Castañón, G. 1998. Respuesta de fertilización y densidad de siembra en líneas de maíz. Agronomía Mesoamericana 9(2):113118.

Vargas, A. 2005. Protocolo de procedimientos de laboratorio: Determinación del nitrógeno foliar. Laboratorio de suelos, plantas, aguas y abonos orgánicos. Instituto Nacional de Innovación y Transferencia en Tecnología Agropecuaria (INTA), Costa Rica. p 4-5.

Villar Zunzunegui, D.F. 2004. Absorción de nutrientes, efecto de la fertilización nitrogenada y potásica y utilización del medidor de clorofila Minolta (modelo SPAD 502) en el manejo del nitrógeno en maíz dulce (Zea mays var. Saccharata). Tesis para optar al grado de Magister en Ciencias Vegetales, Pontificia Universidad Católica de Chile, Facultad de Agronomía e Ingeniería Forestal, Dirección de Investigación y Postgrado, Programa de postgrado en Ciencias de la Agricultura. p 2454. 